\title{
3D Reflectivity Reconstruction by Means of Spatially Distributed Kalman Filters
}

\author{
Gregor F. Schwarzenberg, Uwe Mayer, Nicole V. Ruiter, and Uwe D. Hanebeck
}

\begin{abstract}
In seismic, radar, and sonar imaging the exact determination of the reflectivity distribution is usually intractable so that approximations have to be applied. A method called synthetic aperture focusing technique (SAFT) is typically used for such applications as it provides a fast and simple method to reconstruct (3D) images. Nevertheless, this approach has several drawbacks such as causing image artifacts as well as offering no possibility to model system-specific uncertainties.

In this paper, a statistical approach is derived, which models the region of interest as a probability density function (PDF) representing spatial reflectivity occurrences. To process the nonlinear measurements, the exact PDF is approximated by well-placed Extended Kalman Filters allowing for efficient and robust data processing.

The performance of the proposed method is demonstrated for a 3D ultrasound computer tomograph and comparisons are carried out with the SAFT image reconstruction.
\end{abstract}

\section{INTRODUCTION}

The determination of the reflectivity distribution of a region of interest (ROI) addresses a wide area of applications. Application fields may be found in radar imaging of the earth [1], sonar imaging of the ocean bed [2], seismic imaging of the earth's crust [3] as well as in medical application based on ultrasound imaging systems [4].

The measurement setup for analyzing reflectivity distributions regarded in this paper consists of an arbitrarily distributed sensor network that acquires reflectivity information about a ROI. Additionally, unfocussed transmission of pulses is regarded as this leads to faster data acquisition especially for 3D applications. For each emitter and receiver one data set is acquired, which are then fused to achieve high resolution and high contrast images. By shifting the transducers to different positions, e.g., the movement of an airplane in synthetic aperture radar, the sensing aperture is increased.

The reconstruction of the unknown reflectivity from the measured data is called an inverse problem [5]. The inverse problem refers to the situation of knowing the incidence field of the emitter and the measured data of the receivers and trying to reconstruct the object causing the variation of the incidence field. This requires a precise knowledge of the characteristics of the transducers as well as the physics behind the propagation of the incidence wave and its interaction with the object under investigation. The mathematical

\footnotetext{
Gregor F. Schwarzenberg, Uwe Mayer and Nicole V. Ruiter are with the Institute for Data Processing and Electronics (IPE), Forschungszentrum Karlsruhe, Germany, \{schwarzenberg, mayer, ruiter\}eipe.fzk.de

Uwe D. Hanebeck is with the Intelligent Sensor-Actuator-Systems Laboratory (ISAS), Institute of Computer Science and Engineering, Universität Karlsruhe (TH), Germany, uwe.hanebeck@ieee.org
}

solution of this inverse scattering problem is intractable, thus approximation schemes are applied to yield an analytic [6] or a numerical [7] solution. The solution with the smallest number of approximations is known as diffraction tomography [8], which is, however, computationally expensive as the solution has to be determined iteratively.

To overcome these difficulties, the 3D reflectivity distribution is described by a statistical approach. For this purpose the reflectivity in the ROI is modeled as a probability density function (PDF), which may be of arbitrary shape representing sharp peaks (point scatterers) and structural information about the object under study. The PDF is approximated by spatially well-placed Extended Kalman Filters, each of them estimating a local reflectivity. After the complete data set is processed, the estimates of all Kalman Filters are fused to construct a global reflectivity estimate in an efficient and robust way.

The paper is organized as follows: Section II gives a general overview on the problem of determining the reflectivity distribution measured by an arbitrarily placed sensor network and presents the general key idea. In Section III, the proposed solution is introduced and explained. Section IV presents an application of the proposed image reconstruction method on a 3D ultrasound computer tomograph and compares the results with the usually applied synthetic aperture focusing technique approach.

\section{PROBLEM FORMULATION}

The problem addressed in this paper is an image reconstruction problem. As uncertainties in the overall system and measurement process cause this problem to be ill-posed [9], the solution for the inverse problem is intractable for the considered system setup. In order to render this nonlinear inversion problem tractable, the first-order Born approximation is employed, i.e., the incidence field at each scatterer is assumed to be the only source, neglecting the scattered fields from other scatterers. Furthermore, the refraction of the emitted pulse is ignored.

Each reflection acquired by a receiver is the integral of reflectivity (acoustic impedance, electrical permittivity) over a hypersurface, see Fig. 1.

By intersecting data sets of different receivers, the source can be located if just one reflector is present. The naïve solution would be to calculate the intersection of all ellipsoids. Both analytic and numerical approaches are very timeintensive. Additionally, in the presence of spatial noise or noise in the data preprocessing the intersections are not exact 


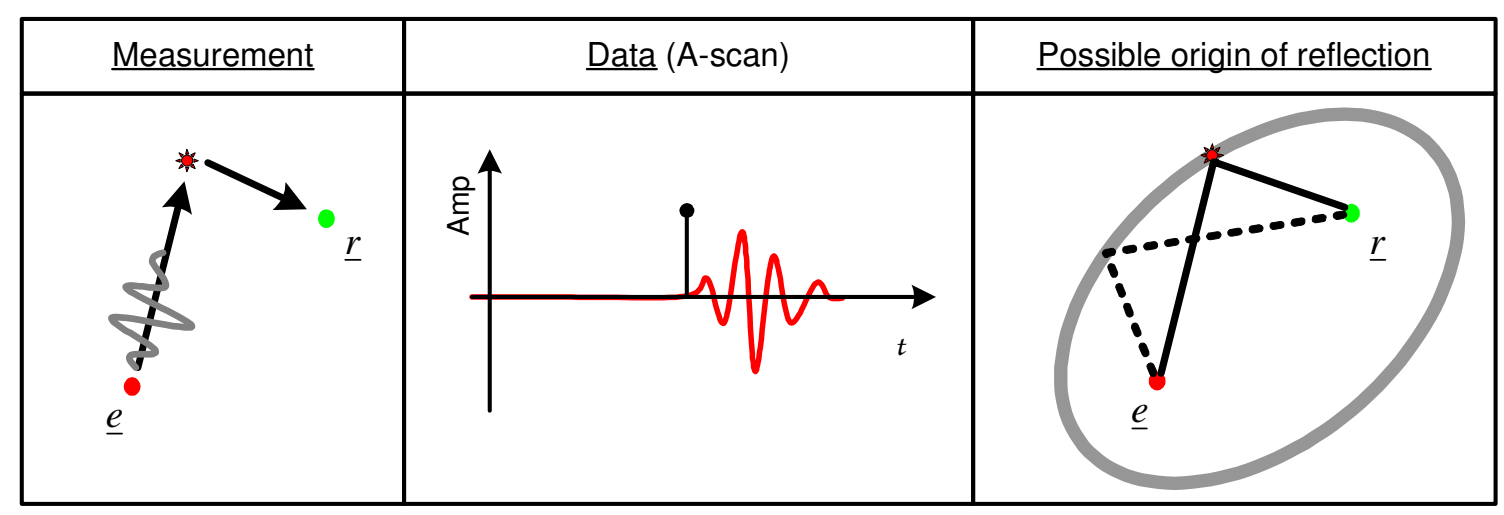

Fig. 1. Measurement and data interpretation: On the left an arbitrarily placed emitter $\underline{e}$ emits a pulse that is reflected by a scatterer and recorded by an arbitrarily placed receiver $\underline{r}$. The measurement of the receiver is plotted in the middle as an amplitude-over-time plot (A-scan). The value of interest is the time-of-arrival as shown by the flag. From only one measurement the exact position of the scatterer can not be derived, only a region containing the scatterer. Assuming a constant speed of propagation of the emitted pulse, this region becomes elliptical with emitter $\underline{e}$ and receiver $\underline{r}$ as focal points, as each position on this ellipse has the same summed distance to emitter and receiver (e.g., the solid and the dashed line). In $3 \mathrm{D}$ this elliptical region becomes a rotation symmetric ellipsoid, more precisely a prolate spheroid.

and statistical or heuristic measures have to be applied to compensate for deviations from the ideal intersection areas.

Since under the Born approximation each measurement restricts the position of the source on a hypersurface in $3 \mathrm{D}$, only a few points on the surface are true scatterer positions. All others may be considered as false-positives and represent themselves as ghosts in the resulting reconstructed reflectivity image. The fusion of several measurements may therefore be interpreted as a data association problem. The complexity class of the corresponding optimal Bayesian solution is NP-hard and while further research in this area is still in progress, the data association problem is not explicitly addressed in this paper.

In addition, real objects do not consist of ideal point scatterers. Regarding non-isotropic scattering, damping, and the characteristics of the finite-sized transducers, reflections from the object are not present in all acquired measurements. This forbids solutions that fuse the measurements in a multiplicative manner, which would cause blindness for directive scatterers.

In order to avoid intersection calculations and regard realistic scattering behavior, the underlying space is sampled to create localized reflectivity estimates. The key idea is to keep track of all true- and false-positive reflections in the sampled volume and to estimate a probability density that denotes for each point the probability of being the source of a reflection. These local estimates enable the consideration of system-specific properties of the measurement process, e.g., regarding sensor characteristics with respect to the location of the estimator. After processing all measurements, these distributed samples are fused to an approximation of the global PDF, which is then used to create an image of reflectivity.

Here the question arises, how those local samples are represented and how the nonlinear measurements (hypersurfaces) are applied to update the PDF. Since the complete data set of the $3 \mathrm{D}$ sensor system is usually too large to be processed altogether, it is of interest to obtain a recursive update formulation. Additionally, due to the numerous error parameters interfering with each other, the overall measurement error is modeled as normal probability density, as stated by the central limit theorem. The Kalman Filter as an optimal recursive filter under the condition of normal distributions for the system model and noise is a good choice for representing the local samples. In order to handle the nonlinearity of the measurement data, the Extended Kalman Filter (EKF) is applied, which linearizes the model equations using a first order Taylor series approximation [10].

\section{REFLECTIVITY PROBABILITY DENSITY}

This section outlines the basic model setup derived to approximate the PDF of a 3D reflectivity distribution from the measurements of a distributed sensor network. At first, the approximation of the exact PDF is introduced, followed by the model equations of the EKF. Then an efficient measurement to filter assignment is presented and the update of the PDF is explained, concluded with the creation of an image based on the PDF.

\section{A. PDF approximation}

The exact PDF is approximated by distributed Extended Kalman Filters that cover the ROI (Fig. 2). Each of them is a local estimator of reflectivity. The state vector of the filter is composed of the 3D position of the reflector and can be extended by any other parameter that may be extracted robustly from the raw input data, e.g., a frequency analysis by means of a short-time Fourier transform of the current echo. These parameters are an additional aid for the improvement of the reflectivity estimate of the object under study.

\section{B. Extended Kalman Filter}

In the following scenario, we assume a discrete-time dynamic system with linear system model, but nonlinear 


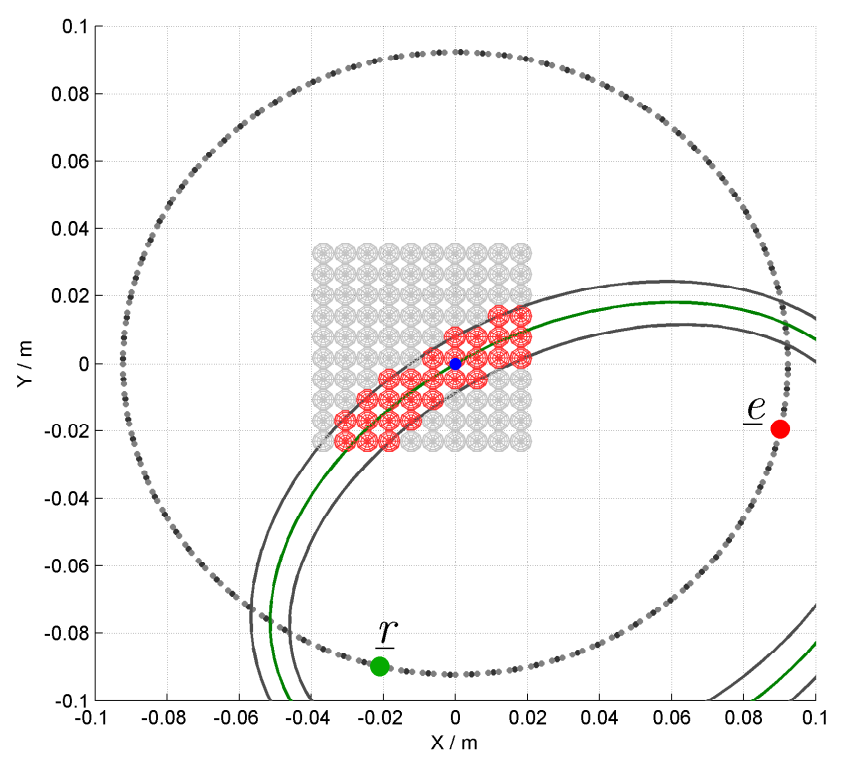

Fig. 2. Example for Kalman Filter placement and affected filters for one measurement. The large dashed circle represents the sensing aperture with one selected emitter $\underline{e}$ and receiver $\underline{r}$. One measurement (time-ofarrival) covers an elliptical region and the measurement noise determines the thickness of this region. The ROI is covered with Kalman Filters whose initial covariances are plotted as spheres with the standard deviation as radius. As shown, the current measurement does only affect a subset of all filters, which is exploited for an efficient measurement update.

measurement model for the local estimates,

$$
\begin{aligned}
\boldsymbol{x}_{\boldsymbol{k}} & =\mathbf{A}_{\mathbf{k}-\mathbf{1}} \boldsymbol{x}_{\boldsymbol{k}-\mathbf{1}}+\boldsymbol{w}_{\boldsymbol{k}-\mathbf{1}}, \\
\boldsymbol{z}_{\boldsymbol{k}} & =h_{k}\left(\boldsymbol{x}_{\boldsymbol{k}}, \boldsymbol{v}_{\boldsymbol{k}}\right), \\
\boldsymbol{w}_{\boldsymbol{k}} & \sim \mathcal{N}\left(0, \mathbf{Q}_{\mathbf{k}}\right), \\
\boldsymbol{v}_{\boldsymbol{k}} & \sim \mathcal{N}\left(0, \mathbf{R}_{\mathbf{k}}\right), \\
\mathrm{E}\left\{v_{k} w_{j}^{T}\right\} & =0 \quad(\forall k \neq j) .
\end{aligned}
$$

Here, $\boldsymbol{x}_{\boldsymbol{k}}$ is a $n \times 1$ state vector at time step $k$, containing at least the $[x, y, z]^{T}$ position of a local reflection. $\mathbf{A}_{\mathbf{k}-\mathbf{1}}$ is the time-variant linear $n \times n$ system matrix, which relates the state vector at time step $k-1$ to time step $k$, disturbed by the system noise $\boldsymbol{w}_{\boldsymbol{k}}$ drawn from a zero mean multivariate normal distribution with covariance $\mathbf{Q}_{\mathbf{k}}$ of size $n \times n$. $z_{k}$ is a $d_{z} \times 1$ measurement vector, which is a scalar if only the time-of-arrival (TOA) is taken into consideration. $h_{k}\left(x_{k}, v_{k}\right)$ is the time-variant, nonlinear measurement function, which returns a $d_{z} \times 1$ estimate of the next measurement, given $\boldsymbol{x}_{\boldsymbol{k}}$ and the zero mean Gaussian white measurement noise $\boldsymbol{v}_{\boldsymbol{k}}$ with covariance $\mathbf{R}_{\mathbf{k}}$ of size $d_{z} \times d_{z}$.

The time update equations of the Kalman Filter are given as [11]

$$
\begin{aligned}
& \boldsymbol{x}_{\boldsymbol{k}}^{-}=\mathbf{A}_{\mathbf{k}-\mathbf{1}} \boldsymbol{x}_{\boldsymbol{k}-\mathbf{1}}^{+}, \\
& \mathbf{P}_{\mathbf{k}}^{-}=\mathbf{A}_{\mathrm{k}-1} \mathbf{P}_{\mathbf{k}-1}^{+} \mathbf{A}_{\mathbf{k}-1}{ }^{T}+\mathbf{Q}_{\mathbf{k}} .
\end{aligned}
$$

Based on the system model $\mathbf{A}_{\mathbf{k - 1}}$, the state $\boldsymbol{x}_{\boldsymbol{k}}^{-}$as well as the estimate covariance $\mathbf{P}_{\mathbf{k}}^{-}$is predicted for the current time step $k$.
The measurement update equations as defined by the Extended Kalman Filter are [10],

$$
\begin{aligned}
\mathbf{S}_{\mathbf{k}} & =\mathbf{H}_{\mathbf{k}} \mathbf{P}_{\mathbf{k}}^{-} \mathbf{H}_{\mathbf{k}}^{T}+\mathbf{R}_{\mathbf{k}}, \\
\mathbf{K}_{\mathbf{k}} & =\mathbf{P}_{\mathbf{k}}^{-} \mathbf{H}_{\mathbf{k}}^{T} \mathbf{S}_{\mathbf{k}}{ }^{-1}, \\
\boldsymbol{x}_{\boldsymbol{k}}^{+} & =\boldsymbol{x}_{\boldsymbol{k}}^{-}+\mathbf{K}_{\mathbf{k}}\left(\boldsymbol{z}_{\boldsymbol{k}}-h_{k}\left(\boldsymbol{x}_{\boldsymbol{k}}^{-}, 0\right)\right), \\
\mathbf{P}_{\mathbf{k}}^{+} & =\left(\mathbf{I}-\mathbf{K}_{\mathbf{k}} \mathbf{H}_{\mathbf{k}}\right) \mathbf{P}_{\mathbf{k}}^{-} .
\end{aligned}
$$

The measurement prediction covariance $\mathbf{S}_{\mathbf{k}}$ is the expected value of the innovation, calculated by means of the predicted covariance $\mathbf{P}_{\mathbf{k}}^{-}$and the linearization $\mathbf{H}_{\mathbf{k}}$ of the measurement function $h_{k}$. The $d_{z} \times n$ Jacobian matrix $\mathbf{H}_{\mathbf{k}}$ is dependent on the predicted state $\boldsymbol{x}_{\boldsymbol{k}}^{-}$,

$$
\mathbf{H}_{\mathbf{k}}=\frac{\partial h_{k}}{\partial \boldsymbol{x}_{\boldsymbol{k}}}\left(\boldsymbol{x}_{\boldsymbol{k}}^{-}, 0\right) .
$$

$\mathbf{K}_{\mathbf{k}}$ is the $n \times d_{z}$ Kalman gain, which is used to update the state estimate to $\boldsymbol{x}_{\boldsymbol{k}}^{+}$and its covariance $\mathbf{P}_{\mathbf{k}}^{+}$.

\section{Measurement to filter assignment}

Each measurement of an emitter-receiver-combination is an amplitude-over-time signal (A-scan). For reflectivity imaging, parameters such as TOA, amplitude, frequency or phase information of each echo need to be extracted in a preprocessing step.

As demonstrated in Fig. 1, each preprocessed echo does only affect a specific volume (ellipsoidal shell) from where the possible scatterer(s) caused the reflection. The size of this volume increases with higher uncertainty of the preprocessing step. Therefore, a filter is only affected if it is close to this volume. This is exploited to achieve an efficient processing of the Kalman Filter updates as only a subset of all filters need to be updated for each measurement.

This is achieved by using one part of the Kalman Filter equations. The quadratic form of the innovation covariance $\mathbf{S}_{\mathbf{k}}$ (equation (3)) may be regarded as a squared norm, weighted according to the filter covariance matrix $\mathbf{P}_{\mathbf{k}}^{-}(\mathrm{Ma}-$ halanobis distance). This statistical distance is used to define a set $\mathcal{S}_{k}$ of Kalman Filters at the positions $\boldsymbol{x}_{\boldsymbol{k}}$ that are updated by the current measurement $z_{k}$,

$$
\begin{aligned}
\tilde{y}_{k} & =\boldsymbol{z}_{\boldsymbol{k}}-h_{k}\left(\boldsymbol{x}_{\boldsymbol{k}}^{-}, 0\right), \\
\mathcal{S}_{k}(\gamma) & =\left\{\boldsymbol{x}_{\boldsymbol{k}}: \tilde{y}_{k}^{T} \mathbf{S}_{\mathbf{k}}^{-\mathbf{1}} \tilde{y}_{k} \leq \gamma\right\} .
\end{aligned}
$$

$\mathcal{S}_{k}(\gamma)$ is $\chi^{2}$ distributed with $d_{z}$ degrees of freedom. $\gamma$ is selected beforehand and kept constant during the application.

An example for this gating procedure is shown in Fig. 2, where the possible origin of one measured reflection for the marked emitter and receiver is shown as elliptic region. The central ellipse represents the current measurement, the bounding ellipses represent the error interval defined by, e.g., measurement noise or the imprecise knowledge of the speed of propagation of the emitted pulse.

\section{Independent Kalman Filters}

Before processing the acquired data for reconstruction, Kalman Filters are placed throughout the ROI at a desired 
resolution. This initializes the state vector of each Kalman Filter.

As each TOA measurement is the integral of reflectivity along an ellipsoidal shell, multiple positions and thereby multiple Kalman Filters are affected by the same data. Nevertheless, in this paper the Kalman Filters are assumed to be independent of each other to avoid the large increase in complexity.

1) Update of the Kalman Filters: The nonlinear measurement function $h_{k}$ (equation (1)) returns the summed travel time of the emitted pulse at the propagation speed $v$ between $\boldsymbol{x}_{k}^{-}$to the emitter $\underline{e}$ and $\boldsymbol{x}_{k}^{-}$to the receiver $\underline{r}$,

$$
h_{k}\left(\boldsymbol{x}_{\boldsymbol{k}}^{-}, 0\right)=\frac{\left\|\boldsymbol{x}_{\boldsymbol{k}}^{-}-\underline{e}\right\|+\left\|\boldsymbol{x}_{\boldsymbol{k}}^{-}-\underline{r}\right\|}{v} .
$$

The Jacobian matrix $\mathbf{H}_{\mathbf{k}}$ of this function as defined in equation (4) equals the normal vector of an ellipsoid through the position $x_{k}^{-}$with the focal points $\underline{e}$ and $\underline{r}$.

The update of the distributed Kalman Filters with a new measurement $z_{k}$ is performed as follows: First, the gating procedure is applied to determine those Kalman Filters that have to be updated (Fig 3(a)). The value of $\gamma$ (equation (5)) is set accordingly so that it represents a reasonable amount of space, from where the measurement $z_{k}$ could have originated. Then $\mathbf{H}_{\mathbf{k}}$ and $h_{k}$ are determined for each Kalman Filter state $x_{k}^{-}$. The update with $z_{k}$ causes the filter state to be shifted along the local normal (defined by $\mathbf{H}_{\mathbf{k}}$ ) on the ellipsoid that is defined by $z_{k}$ and the emitter and receiver position (Fig. 3(b)). The update of the covariance matrices results in smaller eigenvalues along the local normal vector. During the processing of different spatial emitter-receivercombinations, the eigenvalues of the covariance matrices are reduced along different normal vectors (Fig. 3(d)). For example all eigenvalues will become smaller at true scatterer positions if the object under study is surrounded by sensors.

\section{E. Image formation}

After processing all measurements with the distributed Kalman Filters, an image has to be created, that shows high values at positions with a high probability of reflectivity. If a Kalman Filter has been placed at or is close to a true scatterer position, multiple measurements will have been used to update the covariance matrix, resulting in a denser probability mass around the filter position. Integrating this density over a predefined voxel grid with a desired resolution results in high image values at those positions where Kalman Filters with small eigenvalues in their covariance matrices are located.

One voxel of the final image is the sum of the integrals of all Kalman Filters over the volume of the regarded voxel. For speed-up purposes, only those Kalman Filters are regarded for a specific voxel that lie closer than four times the standard deviation corresponding to the covariance matrices.

\section{APPLICATION: UltRASOUND COMPUTER TOMOGRAPH}

The derived method is applied to reconstruct reflectivity images of a 3D ultrasound computer tomograph (USCT) [4],

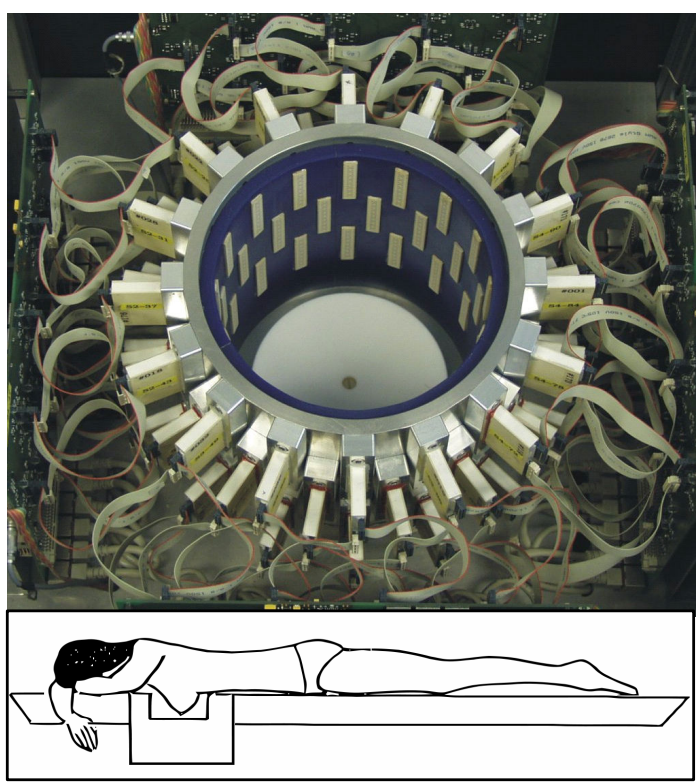

Fig. 4. Experimental ultrasound computer tomograph with cylindrical aperture and approx. 2000 transducers that are grouped into 48 transducer array systems (white blocks). The application is shown at the bottom, the woman lies in prone position on a bed while the breast is hanging in the measurement tank filled with water.

that has been built at the Institute for Data Processing and Electronics at Forschungszentrum Karlsruhe. This system has been developed for early breast cancer diagnosis and enables 3D imaging of a non-deformed breast with non-ionizing radiation. Fig. 4 shows the measurement setup.

The sensing aperture is cylindrical with a height of $15 \mathrm{~cm}$ and a diameter of $18 \mathrm{~cm}$. It is equipped with 384 emitters and 1536 receivers grouped in 48 transducer array systems. The transducers have a size of $(1.4 \mathrm{~mm})^{2}$, a center frequency of $2.4 \mathrm{MHz}$ and an opening angle of \pm 15 degree at $-6 \mathrm{~dB}$. A complete measurement results in a data set of approx. $600.000 \mathrm{~A}$-scans (3 GB), which can be additionally increased by rotating the cylindrical aperture, thereby acquiring more information from different angles.

In this paper, a constant speed of sound is assumed. In future work, varying speed of sound can be introduced by including the speed of sound map of the ROI, which can be determined with the same data set [12].

\section{A. Data preprocessing}

The TOA of each recorded echo is detected by means of a pulse detection method based on the wavelet transform [13]. Each TOA is used as a measurement for updating the distributed Kalman Filters. The ability of the pulse detection to separate two interfering echoes has been evaluated as $900 \mathrm{~ns}$ (center frequency of $2.4 \mathrm{MHz}$ ), which is used as basic magnitude of the measurement noise.

\section{B. Kalman Filter setup}

For the following evaluation, the Kalman Filter equation are adapted as follows: 


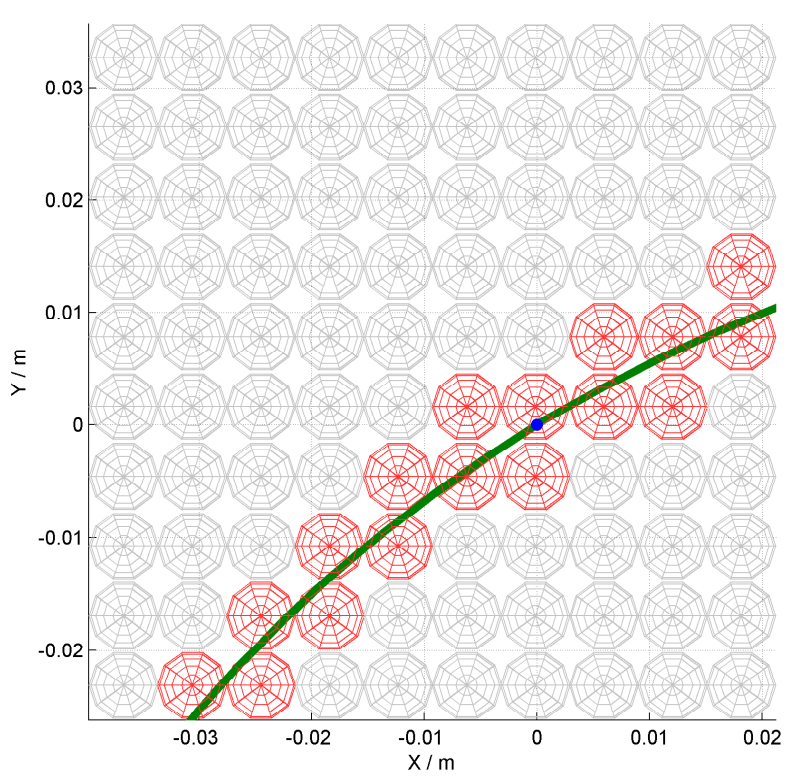

(a) Filter selection (gating)

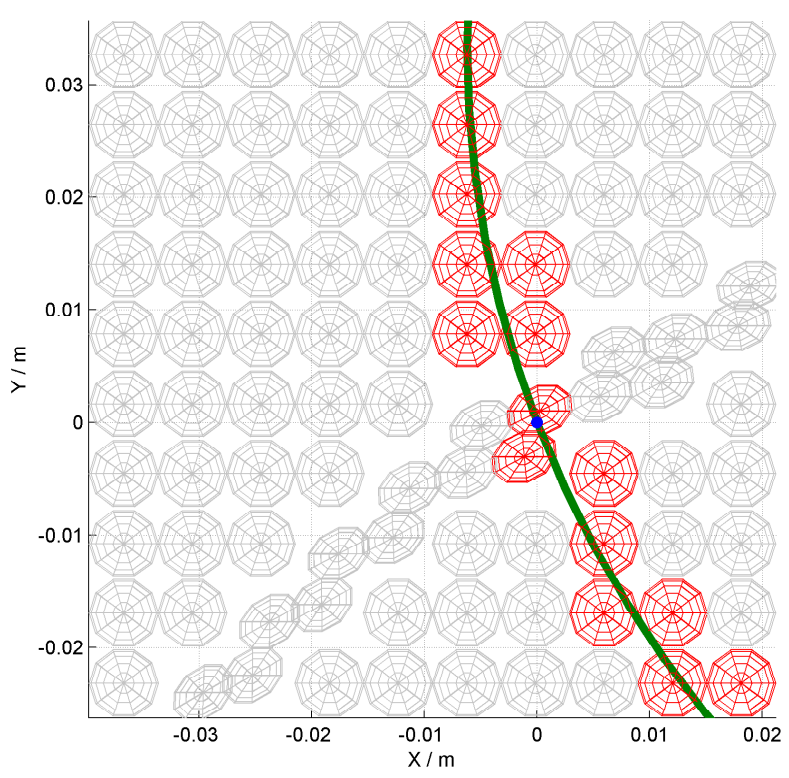

(c) Filter selection (gating)

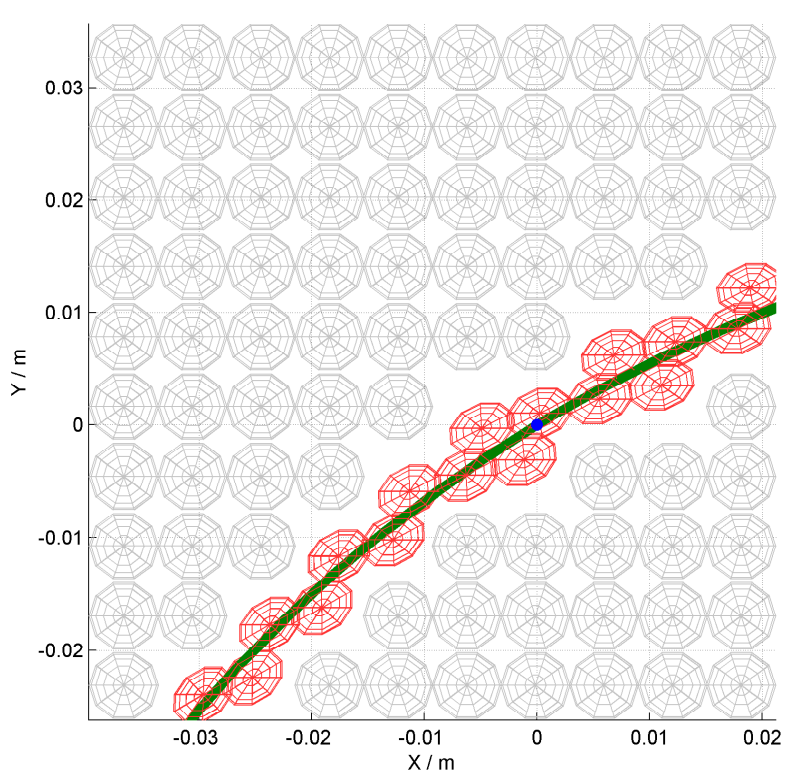

(b) Update of selected filters

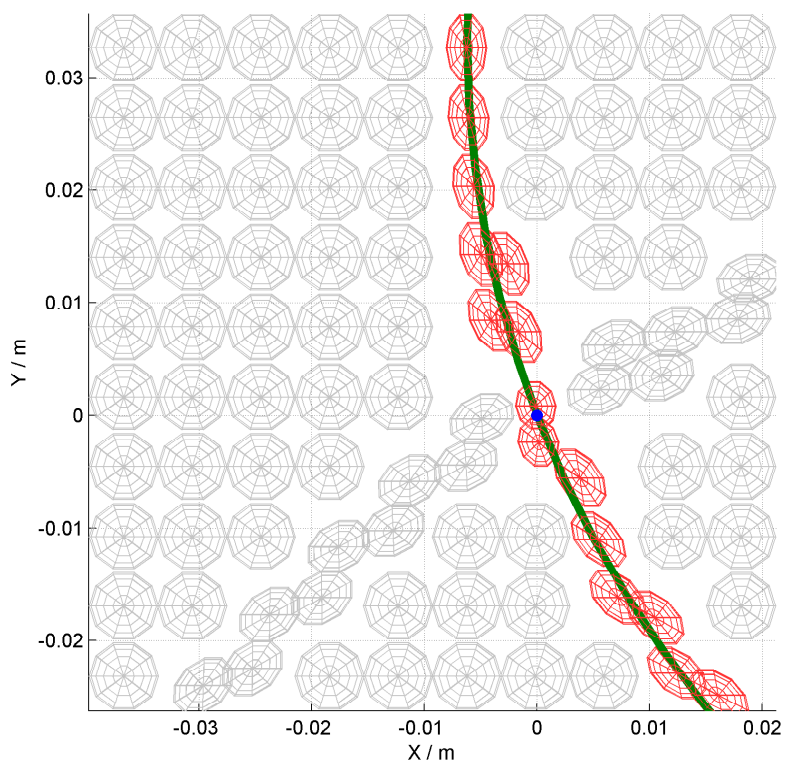

(d) Update of selected filters

Fig. 3. Example of processing two TOA measurements for 100 distributed Kalman Filters. Their covariances are plotted as ellipsoids (initially spheres) centered at the positions of each filter. The point scatterer is shown as blue dot at the position $(\mathrm{x}, \mathrm{y})=(0.0,0.0)$. (a) shows the subset of filters (red) for the first measurement as determined by the gating procedure. The selected filters are updated, which shifts their positions towards the measurement and adapts their covariance matrices accordingly ((b)). The update procedure is shown for a second measurement ((c) and (d)), which causes the covariance of the filter closest to the scatterer to shrink the most. 

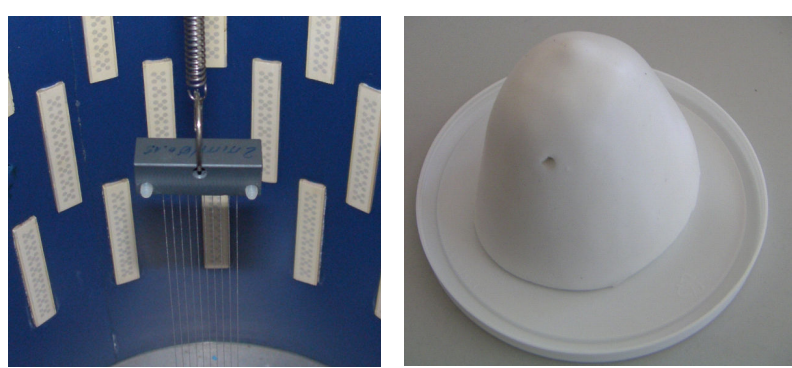

Fig. 5. Left: Ten vertically spanned nylon threads with a diameter of $0.2 \mathrm{~mm}$, each spaced $2 \mathrm{~mm}$ apart for evaluating the horizontal resolution of the USCT. Right: Tissue mimicking triple biopsy breast phantom for evaluating the breast imaging capability of the USCT.

The system model $\mathbf{A}_{\mathbf{k}}$ is set to identity as no prediction on the position of the state vector may be performed. The application of system noise is also neglected. The system noise would increase the eigenvalues of the covariance matrices and thereby unsharpen the image. The analysis of a useful application of system noise is part of future work.

The measurement noise is constant during the training process and has to be set depending on the amount of data and the initial values of the covariance matrices of the Kalman Filters.

The placement of the filters and their initialization is application-dependent. The choice of the number of Kalman Filters and their distribution is an empirical process so far and is based on the desired resolution in the final image or its desired quality with respect to structural information.

\section{Evaluation}

The proposed method is evaluated by means of two experiments. Image reconstructions with the PDF are compared to the currently applied image reconstruction, which is based on synthetic aperture focusing technique (SAFT) [14].

The first experiment consists of ten vertically spanned nylon threads (Fig. 5 left). This experiment was used for experimental resolution assessment in the horizontal plane. The reconstructed images of the nylon threads are compared by evaluating the contrast of each image. A second and more complex experiment with a clinical breast phantom (Fig. 5 right) is done to demonstrate the proposed method for breast imaging.

To reconstruct an image with SAFT, each recorded A-scan $A_{(i, j)}$ from an emitter and a receiver at the positions $\underline{e}_{i}$ and $\underline{r}_{j}$ is backprojected to the image position $\underline{x}$ of the image $I$ using

$$
I(\underline{x})=\sum_{i=1}^{m} \sum_{j=1}^{n} A_{(i, j)}\left(\frac{\left\|\underline{e}_{i}-\underline{x}\right\|+\left\|\underline{r}_{j}-\underline{x}\right\|}{v}\right),
$$

where $v$ is the speed of propagation of the emitted pulse, here assumed to be constant. The A-scans used for the image reconstruction with SAFT are created by convolving the TOA data from the preprocessing step with a Gaussian window of a temporal length of $1 \mu \mathrm{s}$. This compensates for the error induced by the preprocessing step and further errors caused by the imprecise knowledge of the speed of sound and the positioning of the transducers, respectively.

As measure for the contrast, the signal difference to noise ratio (SDNR) is evaluated, which is calculated by comparing the mean amplitude of the reconstructed object $\mu_{\text {object }}$ to the background artifacts. These are evaluated as the mean $\mu_{B G}$ and standard deviation $\sigma_{B G}$,

$$
S D N R=\frac{\mu_{\text {object }}-\mu_{B G}}{\sigma_{B G}} .
$$

For evaluating the contrast of the nylon thread reconstructions, the background has to be segmented from the object. This is performed separately for each nylon thread by taking those pixel into account that have higher values than half of the local maximum.

1) Thread experiment: The ten nylon threads have a diameter of $0.2 \mathrm{~mm}$, each spaced $2 \mathrm{~mm}$ apart and are vertically spanned through the center of the USCT. Only the physically neighboring transducers closest to the slice image were used resulting in 16 sending and 64 receiving elements. This is sufficient for reconstructing the threads but also causes image artifacts due to the sparseness of the sensing aperture. This gives a good basis for comparing the two image reconstruction approaches via the contrast function. The ideal image reconstruction of these threads would result in ten distinct dots as shown at the top of Fig. 6.

The reconstruction of the nylon threads with the SAFT approach $(407 \times 407$ pixel $)$ is shown on the left of Fig. 6 . The ten threads are clearly visible, nevertheless, there are many artifacts in the proximity of the threads. The computed SDNR resulted in a value of 5.1.

The image reconstruction with $87 \times 87$ distributed Kalman Filters is shown on the right of Fig. 6. The threads are also imaged as distinctive points, but the reconstruction artifacts are significantly reduced. The evaluated contrast value of 10.5 is twice times higher than the value of the SDNR of the SAFT image reconstruction.

2) Breast phantom experiment: The clinical breast phantom is a triple modality test object for biopsy and can be imaged with X-ray, MRI, and ultrasound [15]. This breast phantom has several inclusions mimicking cysts and cancer structures. The average attenuation is $0.5 \mathrm{~dB} / \mathrm{MHz} / \mathrm{cm}$. For comparison, a slice region was chosen that shows two cysts that have a strong directive scattering behavior and one cancerous structure that scatters more isotropic.

For this experiment the ground truth for ultrasonic reflectivity is unknown. In order to get an idea of the inner structure of the breast phantom, an MRI image of the same breast phantom has been acquired. The according slice region is shown at the top of Fig. 7. A high resolution reconstruction with SAFT $(805 \times 605$ pixel $)$ is shown on the left of Fig. 7. The boundaries of the cysts are not completely visible and the cancerous structure dominates the image, as most of the backprojections fell in this region. The image reconstruction with $73 \times 55$ Kalman Filters of the same region is shown on the right of Fig. 7. The boundaries of the cysts are more distinct and the cancerous structure does 


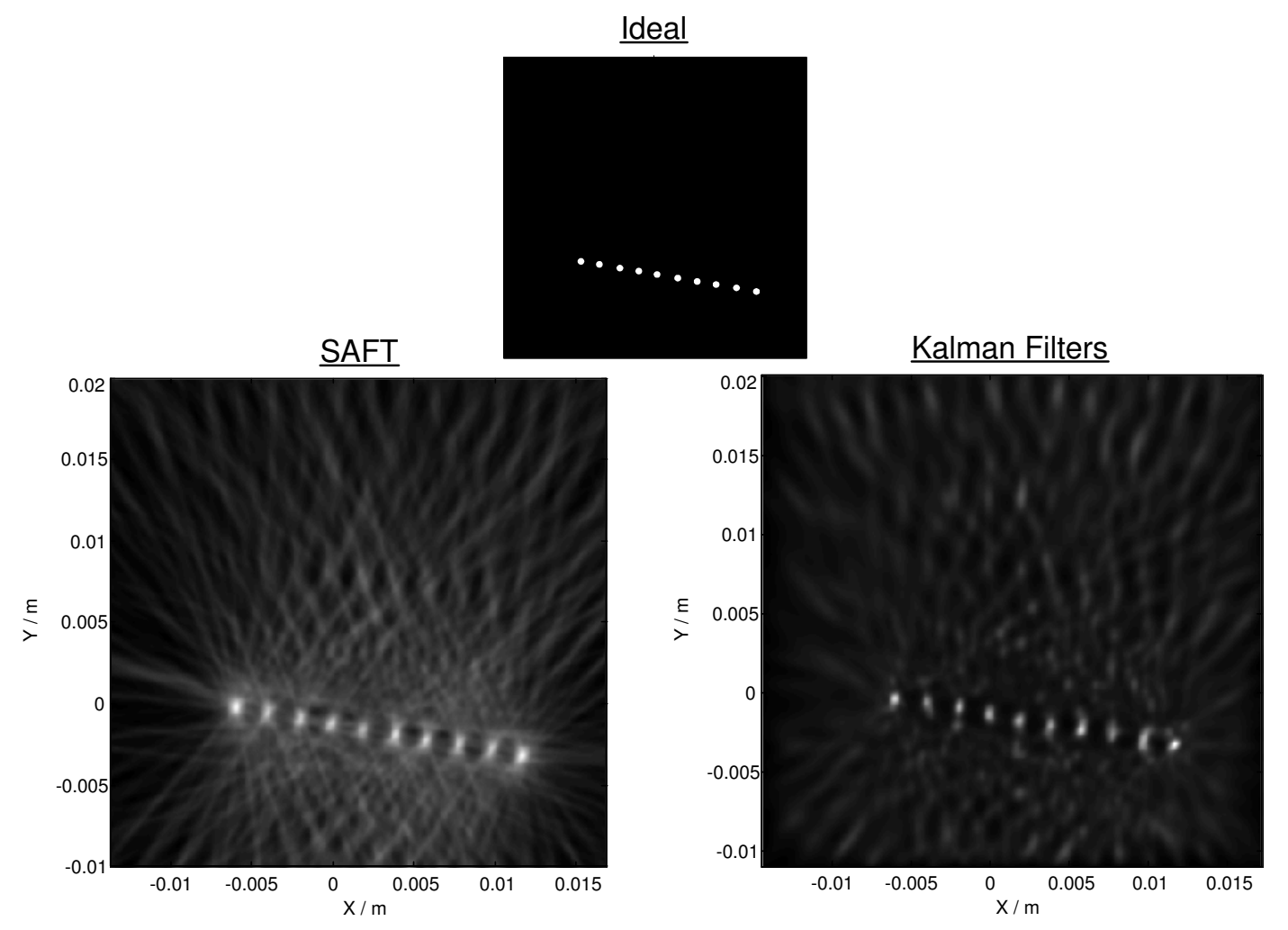

Fig. 6. The slice image reconstruction $(407 \times 407$ pixel $)$ of the ten vertically spanned nylon threads with SAFT shows ten clearly distinguishable points, see the ideal image at the top. Nevertheless, along the normal of the line connecting the ten threads, lots of artifacts are visible, which is due to the sparseness of our sensor aperture. The image reconstruction with $87 \times 87$ distributed Kalman Filters also clearly shows ten points. There are also reconstruction artifacts but less visible and more homogeneous. The SDNR value of this reconstruction is twice times higher compared to the SAFT image reconstruction.

not dominate the image. The high amount of reflections from this region causes the covariance matrices to shrink resulting in a slightly visible grid. Compared to the boundaries of the cysts, which are formed by covariance matrices deformed along the tangent of the boundary, the value calculated from a single covariance matrix is not as high as the sum of largely overlapping Kalman Filters along the boundaries.

\section{COnClusion And Future Work}

A new approach was presented for the reconstruction of the $3 \mathrm{D}$ reflectivity distribution of a region of interest measured by an arbitrarily distributed sensor network. The imaging system regarded here is based on the synthetic aperture approach, which is widely applied in seismic, sonar, and radar imaging. The presented approach models the region of interest as a PDF representing spatial reflectivity occurrences. The data is processed in a recursive manner to update the distributed Extended Kalman Filters used to approximate the PDF. This allows to process the nonlinear measurements as well as fusing information of objects under study that are only partly available in the acquired measurements.

Experiments with a 3D ultrasound computer tomograph showed that the proposed method results in a higher image quality with less image artifacts and higher structural information. The run-time for reconstructing images with the distributed Kalman Filters is in the same order of magnitude as the SAFT approach. The results also showed that the amount of Kalman Filters does not have to be as high as the number of voxel used with the SAFT approach.

The image quality may be additionally improved by regarding system-specific parameters such as sensor characteristics, uncertainties in transducer positioning, and speed of propagation determination as well as object properties. With the proposed method, a basic framework is available for future work. More precisely, the following issues will be analyzed:

- The estimation of measurement noise during Kalman Filter training with a second estimation of the reflectivity may help reduce the artifacts further and also sharpen the image.

- The application of system noise to those Kalman Filters that were not affected by processing the TOA data of one A-scan could also decrease false-positives.

- The utilization of the information of non-occurring echoes at specific times could eventually be used to diminish directly artifacts in the image.

This work demonstrated a statistical approach for 3D image reconstruction, which is easily extendable with systemspecific parameters and is able to consider uncertainties in system parameters and input data. 


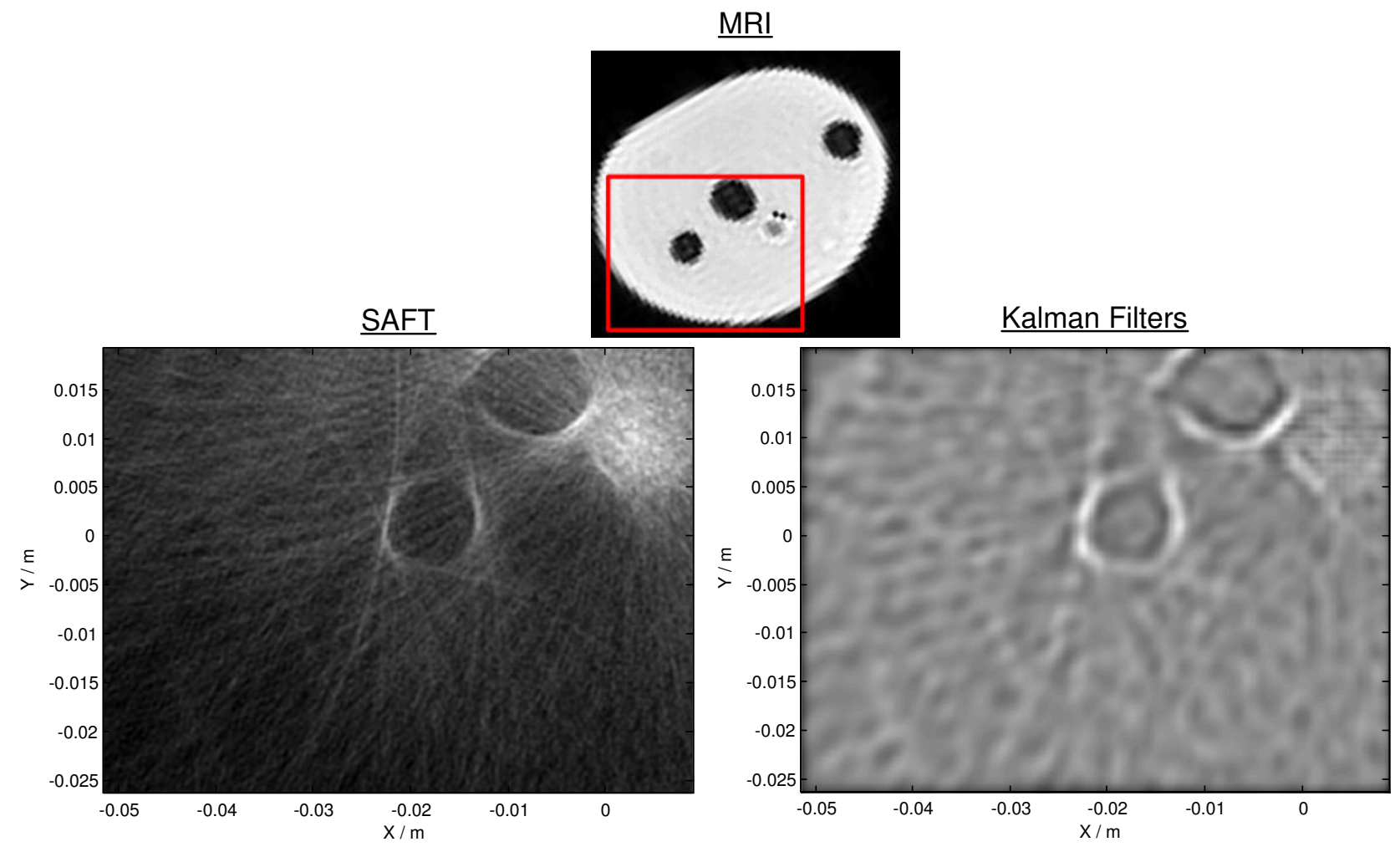

Fig. 7. The slice image reconstruction $(805 \times 605$ pixel $)$ of the breast phantom with SAFT shows two cyst mimicking structure that have a directive scattering behavior as well as another circular structure ("cancer") that has scatters more isotropic. For comparison, see the MRI image of the whole slice at the top with the marked region chosen for evaluation. The boundaries of the cysts are not completely visible and the strong scattering region dominates the image as a bright region. The image reconstruction with $73 \times 55$ distributed Kalman Filters shows the boundaries of the cysts more clearly. The cancerous region is displayed completely different compared to the SAFT image. The high amount of reflections from this area caused the covariance matrices to shrink, which is slightly visible as dot grid. Due to the reduced data set taken for this reconstruction, the skin of the breast is not visible in the ultrasound images, as the skin reflects the ultrasonic pulses to regions that are far below the regarded slice image.

\section{ACKNOWLEDGMENTS}

The financial support of the German Research Foundation (DFG) is gratefully acknowledged. Thanks also to the reviewers for their comments.

\section{REFERENCES}

[1] S. A. Hovanessian, Introduction to Synthetic Array and Imaging Radar. Artech House, 1980

[2] J. Chatillon and M. Zakharia, "Synthetic aperture sonar for seabed imaging: Relative merits of narrow-band and wideband approaches," IEEE Journal of Oceanic Engineering, vol. 17, no. 1, pp. 95-105, 1992.

[3] J. F. Claerbout, Earth soundings analysis: Processing versus inversion. Blackwell Scientific Publications, Inc., 1992.

[4] H. Gemmeke and N. V. Ruiter, "3D ultrasound computer tomography for medical imaging," Nuclear Instruments and Methods in Physics, vol. 580, no. 2, pp. 1057-1065, 2007.

[5] University of Alabama, "Inverse problems." [Online]. Available: http://www.me.ua.edu/inverse/

[6] S. J. Norton and M. Linzer, "Ultrasonic reflectivity imaging in three dimensions: Exact inverse scattering solutions for plane, cylindrical and spherical apertures," in IEEE Transactions on Biomedical Engineering, vol. 28 , no. 2, 1981, pp. 202-220.

[7] — - "Ultrasonic reflectivity imaging in three dimensions: Reconstruction with spherical transducer arrays," in Ultrasonic Imaging, vol. 1 , no. 1,1979 , pp. 210-231.

[8] K. W. A. van Dongen and W. M. D. Wright, "A forward model and conjugate gradient inversion technique for low-frequency ultrasonic imaging," Journal of the Acoustical Society of America, vol. 120, no. 4, pp. 2086-2095, 2006.
[9] F. Lingvall, "Time-domain reconstruction methods for ultrasonic array imaging - a statistical approach,” Ph.D. dissertation, Uppsala University, 2004.

[10] Y. Bar-Shalom and T. E. Fortmann, Tracking and Data Association. Academic Press, Inc., 1988.

[11] R. E. Kalman, "A new approach to linear filtering and prediction problems," Transactions of the ASME, Journal of Basic Engineering, vol. 580, no. 82, pp. 35-45, 1960.

[12] N. V. Ruiter, R. Schnell, M. Zapf, J. Kissel and H. Gemmeke, "Phase aberration correction for 3D ultrasound computer tomography images," in IEEE Ultrasonics Symposium, New York, NY, USA, Oct. 2007, pp. 1808-1811.

[13] G. F. Schwarzenberg, M. Weber, T. Hopp and N. V. Ruiter, "Modelbased pulse detection for 3D ultrasound computer tomography," in IEEE Ultrasonics Symposium, New York, NY, USA, Oct. 2007, pp. $1255-1258$.

[14] K. Mayer, R. Marklein, K. J. Langenberg and T. Kreutter, "ThreeDimensional Imaging System Based on Fourier Transform Synthetic Aperture Focusing Technique," in Ultrasonics, vol. 28, no. 4, 1990, pp. 241-255.

[15] CIRS Incorporated, "Tissue simulation \& phantom technology," Norfolk, VA, USA. 